\title{
Obesity and Related Chronic Health Conditions as Predictors of Motivation to Engage in Healthy Eating Behaviors Among Black Adults
}

\author{
Carolyn M. Tucker • Tasia M. Smith • Tya M. Arthur • \\ Whitney Wall
}

Received: 29 October 2013 /Revised: 28 February 2014 / Accepted: 31 March 2014 / Published online: 7 May 2014

(C) W. Montague Cobb-NMA Health Institute 2014

\begin{abstract}
Introduction Blacks in the USA have a high prevalence of obesity and therefore suffer disproportionately from obesityrelated chronic health conditions. Eating a poor diet is a major contributor to obesity and obesity-related chronic health conditions (e.g., heart disease, hypertension, type 2 diabetes) in Blacks. Having medical concerns has been identified in earlier research to be a motivator to eat a healthy diet among Blacks. Purpose The purpose of this study was to examine body mass index (BMI) as a predictor of motivation to eat healthy due to medical concerns and to determine whether this relationship is mediated by reported number of chronic health conditions. Methods A cross-sectional sample of 207 Black adults (45.9\% males; age: $M=38, \mathrm{SD}=14.12$; $\mathrm{BMI}: M=27.56, \mathrm{SD}=6.55)$ in Bronx, New York, completed questionnaires (i.e., the Motivators of and Barriers to Health-Smart Behaviors Inventory and a Demographic and Health Information Data Questionnaire) at a variety of community-based sites in this city, including hospitals, laundry mats, and street locations.

Results A mediation model was tested using Preacher and Hayes' simple mediation macro for SPSS. BMI was not a significant direct predictor of motivation to eat healthy; however, BMI significantly predicted motivation to eat healthy indirectly through reported number of chronic health conditions.

Conclusion Interventions developed by health promotion and health disparities researchers to increase motivation to eat healthy should consider increasing awareness/knowledge of health risks associated with obesity and related chronic health
\end{abstract}

C. M. Tucker • T. M. Smith $(\bowtie) \cdot$ T. M. Arthur • W. Wall

Department of Psychology, University of Florida, P.O. Box 112250,

Gainesville, FL 32611-2250, USA

e-mail: tmsmith4@ufl.edu conditions and promoting routine health care visits to facilitate early diagnoses of chronic health conditions as integral intervention components.

Keywords Obesity disparities $\cdot$ Motivation $\cdot$ Healthy eating · Chronic health conditions

\section{Introduction}

Blacks typically consume a diet that (a) is deficient in fruits, vegetables, and whole grains; (b) contains excessive amounts of meats, saturated fat, and sodium; and (c) is less healthy overall compared to the diet of other racial/ethnic groups [1-3]. This diet greatly contributes to the alarming rates of obesity in Black adults [4]. Specifically, more than three out of four Black adults are overweight or obese [5]. This prevalence rate is higher for Black adults than all other racial/ethnic groups [5].

The prevalence of obesity among Blacks is particularly troubling given that obesity has a strong association with the occurrence of chronic health conditions - an association similar to the effect of 20 years of aging on chronic health conditions [6]. The chronic health conditions associated with obesity include conditions such as cardiovascular disease, stroke, hypertension, type 2 diabetes, and certain forms of cancer [7]. These health conditions represent four of the six leading causes of death in the USA [8].

Much like obesity, poor diet is a well-established major contributor to most chronic health conditions [9]. An estimated 1,000 deaths per day can be attributed to poor diet and low levels of physical activity [10,11]. Furthermore, when compared to non-Hispanic Whites, Blacks experience higher rates of morbidity and mortality due to chronic health conditions (e.g., hypertension and type 2 diabetes) [12]. 
Health-promoting behaviors, particularly eating behaviors, are influenced by interactions between cognitive/personal and social/environmental determinants [13]. Although important to health behavior, social/environmental determinants (e.g., access to neighborhood food stores) are often intractable. Thus, there is an increasing focus on empowerment theories to guide interventions to foster health-promoting behaviors, such as increasing fruit and vegetable consumption and reducing and/or eliminating health disparities [14, 15]. One such theory is the culturally sensitive Health Self-Empowerment (HSE) Theory.

According to the HSE Theory, self-empowermentoriented, cognitive-behavioral variables are key to understanding and changing health behaviors such as eating behaviors among culturally diverse groups $[16,17]$. The components of the HSE Theory are five literature-based, individually controllable, modifiable variables: (a) health self-efficacy, (b) health motivation, (c) self-praise of health-promoting behaviors, (d) health responsibility/knowledge, and (e) active coping strategies for managing stress and depression [18].

Among the HSE Theory variables, motivation likely influences the act of engaging in health-promoting behaviors the most [18]. Motivation is defined as an intrinsic process, impulse, or intention that moves an individual to the point of readiness to act [19]. Motivators are intrinsic or extrinsic stimulators that jump-start the motivation process, drive individuals to overcome barriers, and increase the benefits of an action. Research has shown that individuals are not all motivated by the same motivators to engage in health-promoting behaviors [20].

Identifying motivators that will impel the motivational process among Blacks is important, since a lack of motivation is a major cause for less than optimal engagement in healthpromoting behaviors like healthy eating. The Health Belief Model posits that individuals who perceive themselves to be at risk for illness or the negative consequences of illness are more likely to engage in health-promoting behaviors than those who do not see themselves to be at risk [21]. Moreover, Rogers' Protection Motivation Theory states that perceived risk and severity of illness influence motivation to engage in health-promoting behaviors, which in turn influences actual behavior [22].

Given the focus of the media on the links between obesity and chronic health conditions and on the importance of a healthy diet to overcome obesity, it is likely that some individuals who are overweight or obese are motivated to eat healthy due to medical concerns. However, there are some individuals who do not appear to have such motivation. It is possible that the actual number of chronic health conditions that one experiences influences the linkage between overweight/obesity and motivation to eat healthy due to medical concerns.

The purpose of this study was to examine body mass index (BMI) as a predictor of motivation to eat healthy due to medical concerns among Black adults in Bronx, New York. Additionally, this study examined the indirect effect of the reported number of chronic health conditions on the relationship between BMI and motivation to eat healthy due to medical concerns among these adults. To our knowledge, no studies to date have examined these relationships.

The following hypotheses were tested in the present study involving Black adults in the Bronx as participants: (a) BMI is a significant positive predictor of motivation to eat healthy due to medical concerns among the Black adults participating in this study (hypothesis 1) and (b) this relationship will be partially mediated by reported number of chronic health conditions (hypothesis 2). The following research question was also explored: Are socio-demographic variables (i.e., age, gender, and household income) associated with BMI, number of reported chronic health conditions, and motivation to eat healthy due to medical concerns among the study participants?

\section{Methods}

\section{Participants}

The cross-sectional sample consisted of 207 Black adult participants. These participants identified as African American (69.6\%), African American of African descent (5.3\%), African American of Caribbean descent (7.7\%), African (4.8\%), Caribbean (10.1\%), and Black-other (2.5\%). Black Hispanics $(n=108)$ were excluded from the current study. Inclusion criteria were being aged 18 years or older, able to read and respond in English, and physically being in Bronx, New York, at the time of participation in the study. The Bronx has one of the highest rates of obesity among the five New York City boroughs [23]. The prevalence of obesity and the large number of predominately Black neighborhoods in the Bronx made it an ideal location for this study [24].

The mean age of the participants was 38 years $(\mathrm{SD}=14.12)$ and the majority of the participants were female (54.1\%). The median household income category was $\$ 20,000-\$ 29,999$, and high school or GED was the highest level of education for $43.0 \%$ of the participants. The majority of the participants were overweight or obese $(55.1 \%)$, which suggests that the study sample is representative of the Black population in the Bronx. Demographic characteristics of the study participants are presented in Table 1.

\section{Measures}

Demographic and Health Information Data Questionnaire (DHIDQ) The DHIDQ was constructed by the authors to obtain the following demographic information: (a) age, (b) sex, (c) highest level of education completed, and (d) household 
Table 1 Demographic characteristics of the sample

\begin{tabular}{|c|c|c|}
\hline \multicolumn{3}{|l|}{ Characteristic } \\
\hline & No. & $\%$ \\
\hline \multicolumn{3}{|l|}{ Gender } \\
\hline Female & 112 & 54.1 \\
\hline Male & 94 & 45.4 \\
\hline No response & 1 & 0.5 \\
\hline \multicolumn{3}{|l|}{ Age } \\
\hline $18-24$ & 48 & 23.2 \\
\hline $25-34$ & 52 & 25.1 \\
\hline $35-49$ & 52 & 25.1 \\
\hline 50 or older & 49 & 23.7 \\
\hline No response & 6 & 2.9 \\
\hline \multicolumn{3}{|l|}{ Household income } \\
\hline Less than $\$ 10,000$ & 61 & 29.5 \\
\hline$\$ 10,000-\$ 29,999$ & 48 & 23.2 \\
\hline$\$ 30,000-\$ 49,999$ & 45 & 21.7 \\
\hline$\$ 50,000$ or more & 46 & 22.2 \\
\hline No response & 7 & 3.4 \\
\hline \multicolumn{3}{|l|}{ Education } \\
\hline Less than high school & 10 & 4.8 \\
\hline High school/GED & 89 & 43.0 \\
\hline 2-year college/technical school & 56 & 27.1 \\
\hline College & 31 & 15.0 \\
\hline Graduate/professional school & 12 & 5.8 \\
\hline No response & 9 & 4.3 \\
\hline \multicolumn{3}{|l|}{ Body mass index } \\
\hline Underweight & 6 & 2.9 \\
\hline Normal weight & 74 & 35.7 \\
\hline Overweight & 61 & 29.5 \\
\hline Obese & 53 & 25.6 \\
\hline No response & 13 & 6.3 \\
\hline \multicolumn{3}{|l|}{ Number of chronic health conditions } \\
\hline 0 condition & 131 & 63.3 \\
\hline 1 condition & 54 & 26.1 \\
\hline 2 conditions & 18 & 8.7 \\
\hline 3 conditions & 4 & 1.9 \\
\hline
\end{tabular}

income. The DHIDQ was also used to obtain self-reported number of chronic health conditions (i.e., Do you currently have any of the following health conditions? (Mark all that apply) (a) high cholesterol, (b) overweight/obesity, (c) heart disease, (d) cancer, (e) hypertension, (f) type 2 diabetes, and ( $g$ ) other chronic health conditions). Number of chronic health conditions was calculated as the sum of all chronic health conditions reported except for "overweight/obesity." The DHIDQ was also used to obtain self-reported BMI (i.e., selfreported height and weight, which were used to calculate selfreported BMI by dividing weight in pounds by height in inches squared and multiplying by a conversion factor of 703) [25].
Motivators of and Barriers to Health-Smart Behaviors Inventory (MB-HSBI) - Adult Version The MB-HSBI-Adult Version [18] is a 127 -item inventory that measures various motivators and barriers associated with engaging in healthsmart (health-promoting) behaviors. The MB-HSBI is comprised of 4 health-smart behavior domains (i.e., healthy breakfast, healthy foods and snacks, healthy drinks, and physical activity), 8 scales (i.e., a separate motivator scale and barrier scale for each domain), 16 motivator subscales (e.g., availability, convenience), and 12 barrier subscales (e.g., knowledge, social influences). The present study only used the medical issues subscale of the healthy foods and snacks motivator scale (i.e., motivation to eat healthy due to medical concerns). The following is a sample item from the medical issues subscale of the healthy food and snacks motivator scale: When I eat healthy foods (like fruits, vegetables, and lower calorie snacks), it is because... I am concerned about preventing high blood pressure.

Instructions on the MB-HSBI are to rate level of agreement with each item using a four-point Likert scale ranging from 1 (strongly disagree) to 4 (strongly agree). Scale and subscale scores for the MB-HSBI are obtained by calculating the mean of all item responses for each scale or subscale. Higher mean scores on the barrier scales or subscales and on the motivator scales or subscales indicate higher levels of barriers and motivators, respectively, with regard to engaging in healthsmart behaviors. In the current sample, the internal reliability (i.e., Cronbach's alpha) for the four items of the medical issues subscale was 0.71 .

\section{Procedure}

After the University of Florida Institutional Review Board approval was obtained, the following three study phases were implemented:

Phase 1. Training of the Supervisor of Data Collection A university-based faculty member was identified to serve as the data collection supervisor. The responsibilities of this supervisor included (1) identifying, training, and supervising seven culturally diverse data collectors (i.e., graduate students at the university where the data collection supervisor works) to recruit and collect data from study participants; (2) managing data collected and other study-related materials; (3) coordinating the payment of data collectors and participants; and (4) documenting any study procedure deviations or adverse events. The data collection supervisor was trained by the researchers to execute his roles/responsibilities and to do so in a culturally sensitive manner.

Examples of culturally sensitive behaviors in the present study are (a) addressing all potential participants as Mr., Ms., or another title of respect unless otherwise requested; (b) using materials that are no higher than a fifth-grade reading level; (c) 
using informal language that is easy to understand when collecting data from study participants; and (d) offering each participant the option of reading and completing materials with or without assistance.

Phase 2. Recruitment The selected data collectors were trained to recruit at least 300 Black adult participants using zip codes in the Bronx to identify areas that would together increase the likelihood of recruiting fairly equal numbers of Black participants with household incomes less than $\$ 30,000$; between $\$ 30,000$ and $\$ 50,000$; and above $\$ 50,000$. Trained data collectors (1) recruited participants from hospitals, laundry mats, fast food restaurants, work sites, universities, and street locations; (2) explained the purpose of the study, inclusion criteria, and study requirements to potential participants using the recruitment flyer as a recruitment aid; and (3) gave perspective participants a cover letter to read that described the study and explained that no informed consent signature was required due to the anonymity of the study.

Phase 3. Data Collection Participants who self-reported meeting the study inclusion criteria specified on the recruitment flyer and understood the information presented in the cover letter were asked to complete the assessment battery $(\mathrm{AB})$ at the recruitment site and immediately return it to the data collector. Data collectors informed participants that (a) the $\mathrm{AB}$ would take about $30 \mathrm{~min}$ to complete; (b) they should not write their name on the $\mathrm{AB}$; (c) they could receive assistance with reading and completing the $\mathrm{AB}$ from the data collector, if needed; and (d) they will receive a cash payment of $\$ 15$ for their research participation upon completion of the AB. Data collectors returned completed ABs to the supervisor of data collection, who then sent all study-related materials to the principal investigator via secure mail.

\section{Overview of Statistical Analyses}

Prior to conducting the analyses to address the research question and hypotheses set forth in this study, the accuracy of data entry was verified for the variables of interest and these variables were examined for missing data. No imputations were performed for missing data because each variable of interest had less than $15 \%$ missing data. A multiple analysis of variance (MANOVA) was conducted to examine if any significant differences existed between the Black subgroups (i.e., African American, African American of African descent, African American of Caribbean descent, African, Caribbean, and Black-other) on the major study variables. Means and standard deviations of the study variables were computed. Bivariate correlations were performed to explore the research question examining the associations between socio- demographic variables (i.e., age, gender, and household income) and reported (a) BMI, (b) number of chronic health conditions, and (c) motivation to eat healthy due to medical concerns.

Preacher and Hayes' [26] simple mediation macro for SPSS was used to test the hypotheses that (a) BMI is a significant positive predictor of motivation to eat healthy due to medical concerns among the Blacks participating in this study (hypothesis 1) and (b) this relationship will be partially mediated by reported number of chronic health conditions (hypothesis 2). The simple mediation macro uses a non-parametric bootstrapping procedure, which requires no normality assumptions to be met for sampling distributions. In the present study, BMI was entered as the independent variable $(X)$, reported number of chronic health conditions was entered as the mediating variable $(M)$, and motivation to eat healthy due to medical concerns was entered as the outcome variable $(Y)$. Five thousand bootstrap samples were requested, and a $95 \%$ confidence interval was used as the significance criterion for estimate of indirect effects. When the $95 \%$ confidence interval for the estimate of indirect effects does not include zero, as was the case, the suggested statistical significance level is 0.05 [26].

\section{Results}

The overall MANOVA was not significant $(F[5,173]=1.06$, $\mathrm{ns}, \eta 2=0.03$ ), indicating that there were no significant differences between the Black subgroups on any of the major study variables. The mean BMI for the study sample was 27.56 $(\mathrm{SD}=6.55)$, which falls within the overweight range. At least one chronic health condition was reported by $36.7 \%$ of the adult participants, and the participants had a mean score for motivation to eat healthy due to medical concerns of 3.12 $(\mathrm{SD}=0.75$; range $1-4)$.

\section{Test of the Research Question}

Table 2 presents the intercorrelations between sociodemographics (i.e., age, gender, and household income) and (a) self-reported BMI, (b) number of chronic health conditions, and (c) motivation to eat healthy due to medical concerns. Increasing age was significantly associated with greater reported number of chronic health conditions $(r=0.42$, $p<0.01)$ and motivation to eat healthy due to medical concerns $(r=0.33, p<0.01)$. It was also found that being female was significantly associated with higher BMI scores $(r=$ $-0.19, p<0.01)$. Higher household income was significantly associated with lower number of reported chronic health conditions $(r=-0.17, p<0.05)$. It is noteworthy that higher BMI scores were significantly associated with reported number of chronic health conditions $(r=0.26, p<0.01)$, and greater 
Table 2 Intercorrelations between demographic variables, BMI, number of $\mathrm{CHCs}$, and $\mathrm{MC}$-motivation

\begin{tabular}{lllllll}
\hline Variables & 1 & 2 & 3 & 4 & 5 & 6 \\
\hline Age & - & & & & & \\
Gender & $0.20^{* *}$ & - & & & & \\
Household income & -0.09 & 0.02 & - & & & \\
BMI & 0.12 & $-0.19^{* *}$ & -0.03 & - & & \\
Number of CHCs & $0.42^{* *}$ & 0.04 & $-0.17^{*}$ & $0.26^{* *}$ & - & \\
MC-motivation & $0.33^{* *}$ & 0.14 & -0.02 & 0.09 & $0.22^{* *}$ & - \\
\hline
\end{tabular}

$B M I$ body mass index, $M C$-motivation motivation to eat healthy due to medical concerns, $C H C s$ number of chronic health conditions

${ }^{*} p<0.05 ;{ }^{*} p<0.01$

reported number of chronic health conditions was significantly associated with motivation to eat healthy due to medical concerns $(r=0.22, p<0.01)$.

Test of the Hypotheses

Figure 1 presents the path diagram for the mediation model testing the direct effect between BMI and motivation to eat healthy due to medical concerns and the mediated path of number of chronic health conditions. The model explained $6 \%$ of the variance in motivation to eat healthy due to medical concerns. The total effect of BMI on motivation to eat healthy due to medical concerns was not statistically significant $(\beta=$ $0.00, \mathrm{~ns})$; thus, BMI was not a significant predictor of motivation to eat healthy due to medical concerns for the study sample. This finding is contrary to hypothesis 1 . The paths from BMI to reported number of chronic health conditions and from reported number of chronic health conditions to motivation to eat healthy due to medical concerns were both positive and significant $(\beta=0.02, p<0.01$ and $\beta=0.39, p=$ 0.01 , respectively). The test of the mediated path of BMI on motivation to eat healthy due to medical concerns through reported number of chronic health conditions was statistically significant (95\% CI 0.01-0.02, $p<0.05$ ). This finding supports hypothesis 2 (see Table 3 for bootstrap results of the mediation model).

\section{Post Hoc Statistical Power}

A post hoc power analysis was conducted using the $\mathrm{G}^{*}$ Power software [27]. The power analysis was based on the current sample size of 207, using a 2-predictor model at an alpha level of 0.05 . Using the recommended effect sizes by Cohen [28], the post hoc analyses yielded a power of 0.42 for a small effect size $\left(f^{2}=0.02\right)$, which is less than adequate. However, the analyses yielded a power greater than 0.99 for the detection of a medium and large effect size $\left(f^{2}=0.15 ; f^{2}=0.35\right.$, respectively), which is more than adequate.

\section{Discussion}

The present study was conducted to examine if body mass index (BMI) predicts motivation to eat healthy due to medical concerns and if this relationship is mediated by reported number of chronic health conditions among a sample of Black adults in Bronx, New York. Specifically, the hypotheses stated that (a) BMI is a significant positive predictor of motivation to eat healthy due to medical concerns among the Blacks participating in this study (hypothesis 1) and (b) this relationship will be partially mediated by reported number of chronic health conditions (hypothesis 2).

In the present study, BMI was not found to be a significant predictor of motivation to eat healthy due to medical concerns. However, BMI significantly predicted motivation to eat healthy indirectly through reported number of chronic health conditions. In other words, higher BMI scores were associated with a greater reported number of chronic health conditions and reported number of chronic health conditions was positively associated with motivation to eat healthy due to medical concerns. These findings provide support for hypothesis 2 , but not for hypothesis 1 .

The lack of association between BMI and motivation to eat healthy due to medical concerns in this study sample is concerning. A review of the literature suggests that Black adults are less likely to perceive BMI as a threat to health and as a risk factor for developing chronic health conditions

Fig. 1 Estimated simple mediation model

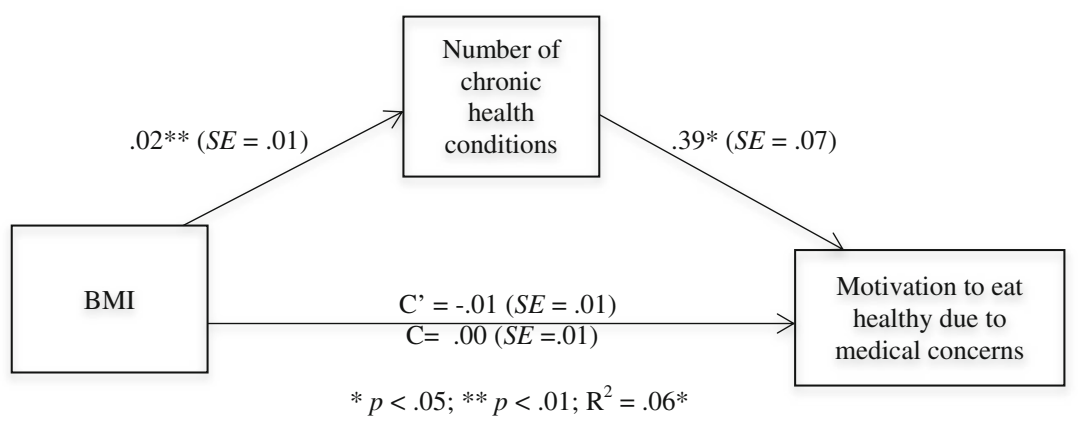


Table 3 Indirect effects of BMI on number of CHCs through MCmotivation

\begin{tabular}{lllll}
\hline Mediator & $\begin{array}{l}\text { Bootstrap } \\
\text { estimate }\end{array}$ & SE & $\begin{array}{l}\text { BCa 95 \% CI } \\
\text { lower }\end{array}$ & $\begin{array}{l}\text { BCa 95 \% CI } \\
\text { upper }\end{array}$ \\
\hline Number of CHCs & 0.01 & 0.00 & 0.01 & 0.02 \\
Total Indirect Effect & 0.01 & 0.00 & 0.01 & 0.02 \\
\hline
\end{tabular}

Based on 5,000 bootstrap samples

$B M I$ body mass index, $M C$-motivation motivation to eat healthy due to medical concerns, $\mathrm{CHCs}$ number of chronic health conditions

when compared to non-Hispanic Whites and Hispanics [29]. This finding in the literature could be partially attributed to the belief in what has been described as "healthy obesity." However, contrary to the belief that an individual can be obese and healthy, findings from a meta-analysis conducted by Kramer and colleagues [30] revealed that obese individuals were at increased risk for long-term, negative health outcomes, even in the absence of metabolic abnormalities (e.g., high blood pressure). Thus, this review of the research suggests that "healthy obesity" is a myth. If overweight/obese Blacks do not perceive their weight as problematic, then they may be less likely to be motivated to engage in healthpromoting behaviors such as healthy eating.

The finding in the present study of a lack of association between BMI and motivation to eat healthy due to medical concerns may be due to a lack of connection between Blacks' perception of what constitutes a healthy weight and the current medical definition of overweight/obese. Blacks are less likely than Whites to perceive themselves as overweight/obese even when they are medically categorized as such [31]. Research also suggests that a larger body size may be perceived more positively in Black communities. Additionally, Black populations have been found to have a lower prevalence of body dissatisfaction when compared to other racial groups, despite having higher rates of overweight and obesity when compared to other racial/ethnic groups in the USA $[32,5]$. Indeed, the differential perception of overweight/obese between Blacks and other racial/ethnic groups may also help explain why BMI was not found to be a predictor of motivation to eat healthy due to medical concerns among the Blacks in the present study.

Another noteworthy finding is that the relationship between BMI and motivation to eat healthy due to medical concerns only emerges when the indirect effect of reported number of chronic health conditions is included in the model. This finding is not surprising given that the prevalence of chronic health conditions, such as hypertension and diabetes, increases as BMI increases from normal weight to obese [5, 33]. Nguyen et al. [34] found that the prevalence of hypertension increases from $18.1 \%$ among normal-weight individuals to $52.3 \%$ among individuals categorized as obese; and the prevalence of diabetes increases from $2.4 \%$ for normal weight to $14.2 \%$ among individuals who are obese.

According to the Health Belief Model [21] and the Protection Motivation Theory [22], individuals who are aware of the negative consequences of illness are more likely to engage in health-promoting behaviors when compared to those who do not see themselves to be at risk for illness. Therefore, Blacks in the present study may be intrinsically motivated by the presence and negative consequences of having a number of chronic health conditions-which increased their motivation to eat healthy due to medical concerns. BMI was not found to be directly associated with motivation to eat healthy; thus, the health risk of obesity may not be a motivator unless Blacks have a number of chronic health conditions.

The findings in the present study highlight the importance of early diagnosis of chronic health conditions among Blacks given that reporting more chronic health conditions was associated with motivation to eat a healthy diet due to medical concerns. These findings also provide support for increasing awareness/knowledge among Blacks that obesity and related chronic health conditions are relevant/important medical concerns. Interventions developed by health promotion and health disparities researchers to increase motivation and healthy eating should consider this awareness/knowledge as an integral intervention component when implementing these interventions with Black adults.

The findings of the exploratory analysis conducted to address the research question are also noteworthy. This question is as follows: Are socio-demographic variables (i.e., age, gender, and household income) associated with BMI, number of reported chronic health conditions, and motivation to eat healthy due to medical concerns among the study participants? One of the findings from investigating this question is that greater age in years was associated with greater reported number of chronic health conditions and motivation to eat healthy due to medical concerns. These findings are supportive of literature suggesting that health disparities among racial/ethnic groups continue into later adulthood and that Blacks continue to suffer from increasing numbers of chronic health conditions [35]. It was also found that household income was associated with lower reported numbers of chronic health conditions, while being female was associated with higher BMI scores. The latter finding is consistent with national statistics suggesting that Black females have higher BMIs than their male counterparts [5].

A strength of this study is that it sought to better understand the relationships among BMI, number of chronic health conditions, and motivation to eat healthy due to medical concerns. Understanding these relationships is important as such understanding has implications for developing programs that may prevent or reduce the occurrence of obesity and related chronic health conditions. Second, this study used the difference 
model research approach — a culturally sensitive research approach that advocates separately studying culturally different groups for the purpose of understanding the group-specific influences on the behaviors of each group rather than simply engaging in the commonly used difference model research approach in which the behaviors of culturally different groups are compared using the behaviors of the majority group as the comparison standard by which the other groups are judged. In the present study, only Black adults were recruited as study participants, which is a research procedure consistent with the difference model research approach [36].

There are notable limitations to this study. One such limitation is that a cross-sectional design was used to collect the data used in this study. This type of design prohibits conclusions regarding causality. In addition, the data for this study were collected using self-report measures. As a result, social desirability bias may be present. It is possible that participants underreported number of chronic health conditions. Additionally, participants could have underreported weight and overreported height, which were both used to calculate BMI in this study. Despite the limitations of self-report measures, these measures are commonly used in health research $[37,38]$. Other limitations of the study were participant recruitment challenges including unpredictability of inclement weather that limited recruitment efforts and inaccessibility of potential Black participants living in unsafe neighborhoods for data collectors to enter. These challenges hindered efforts to recruit the targeted number of participants. However, the number of participants recruited was adequate for the data analyses to test the hypotheses and research question set forth in the present study.

Further research is needed to replicate the findings in this study. Due to the limitations of self-report measures, future studies should consider using clinical data (i.e., clinically measured weight and height to assess BMI and percent body fat) to better account for factors such as muscularity. Future research should also include other important factors that may impact motivation to eat healthy, including access to healthy foods and medical services. Such future research will provide further understanding of the relationships among BMI, number of chronic health conditions, and motivation to eat healthy in Black adults.

Health promotion and health disparities researchers may use this research to inform the development of interventions tailored for the unique needs of Black adults. The results of the present study suggest that such interventions should include a focus on increasing awareness/knowledge of the health risks associated with both overweight/obesity and related chronic health conditions and on promoting routine health care visits that will facilitate early diagnoses of chronic health conditions among Black adults. Given the higher levels of obesity found among the Black females in this study, the suggested interventions may be especially needed among this population.
Acknowledgments Thank you to PepsiCo for providing the grant funding to support the research reported in this paper. The research funder had no involvement in the study design; in the collection, analysis, and interpretation of data; or in the writing of the manuscript.

Informed Consent Statement All procedures followed were in accordance with the ethical standards of the responsible committee on human experimentation (institutional and national) and with the Helsinki Declaration of 1975, as revised in 2000 (5). Informed consent was obtained from all patients for being included in the study.

Animal Rights Statement No animal studies were carried out by the authors of this article.

Conflict of Interest Statement Dr. Carolyn M. Tucker, Ms. Tasia M. Smith, Dr. Tya M. Arthur, and Ms. Whitney Wall declare that they have no conflict of interest.

\section{References}

1. Gary TL, Baptiste-Roberts K, Gregg EW, et al. Fruit, vegetable and fat intake in a population-based sample of African Americans. J Natl Med Assoc. 2004;96(12):1599-605.

2. McCabe-Sellers BJ, Bowman S, Stuff JE, et al. Assessment of diet quality of US adults in the Lower Mississippi Delta. Am J Clin Nutr. 2007;86(3):697-706.

3. U.S. Department of Health and Human Services and U.S. Department of Agriculture. Dietary guidelines for Americans, 2010. Washington, DC: U.S. Government Printing Office. 2010.

4. Sharma S, Cao X, Arcan C, Mattingly M, et al. Assessment of dietary intake in an inner-city African American population and development of a quantitative food frequency questionnaire to highlight foods and nutrients for a nutritional invention. Int J Food Sci Nutr. 2009;60(S5):155-67.

5. Flegal KM, Carroll MD, Kit BK. Prevalence of obesity and trends in the distribution of body mass index among US adults, 1999-2010. J Am Med Assoc. 2012;307(5):491-7. doi:10.1001/jama.2012.39.

6. Sturm R. The effects of obesity, smoking, and drinking on medical problems and costs. Health Aff. 2002;21(2):245-53.

7. Forte V, Pandey A, Abdelmessih R, et al. Obesity, diabetes, the cardiorenal syndrome, and risk for cancer. Cardiorenal Med. 2012;2(2):143-62.

8. Heron M. Deaths: leading causes for 2006. Hyattsville: National Center for Health Statistics; 2010.

9. Roberts CK, Barnard RJ. Effects of exercise and diet on chronic disease. J Appl Physiol. 2005;98:3-30.

10. Mokdad AH, Marks JS, Stroup DF, et al. Actual causes of death in the United States, 2000. J Am Med Assoc. 2004;291(10):1238-45.

11. Mokdad AH. Correction: actual causes of death in the United States, 2000. J Am Med Assoc. 2005;293(3):293.

12. McFayden E. Key factors influencing health disparities among African Americans. Race Gender Class. 2009;16(3-4):120-32.

13. Bandura A. Social foundations of thought and action: a social cognitive theory. Prentice Hall: Englewood Cliffs, NJ; 1986.

14. Tucker CM, Arthur TM, Roncoroni J, et al. Empowering communities for health promotion. In: Johnson C, Diaz J, Franco Z, Friedman H, Nastasi B, editors. Praeger Handbook for Social Justice and Psychology.

15. Tucker CM, Arthur TM, Roncoroni J, et al. Patient-centered culturally sensitive health care. Am J Lifestyle Med. 2013. doi:10.1177/ 1559827613498065.

16. Tucker CM, Butler AM, Loyuk IS, et al. Predictors of a healthpromoting lifestyle and behaviors among low-income African American mothers and white mothers of chronically ill children. $\mathrm{J}$ Natl Med Assoc. 2009;101(2):103-10. 
17. Tucker CM, Daly KD, Herman K. Customized multicultural health counseling: bridging the gap between mental and physical health for racial and ethnic minorities. In: Ponterotto J, Casas M, Suzuki L, Alexander C, editors. Handbook of multicultural counseling. 3rd ed. Thousand Oaks: Sage Publications; 2010.

18. Tucker CM, Rice KG, Hou W, et al. Development of the Motivators of and Barriers to Health-Smart Behaviors Inventory. Psychol Assess. 2011;23(2):487-503. doi:10.1037/a0022299.

19. Coburn CL, Weismuller PC. Asian motivators for health promotion. $\mathrm{J}$ Transcult Nurs. 2012;23(2):205-14. doi:10.1177/1043659611 433869.

20. Carter KF, Kulbok PA. Motivation for health behaviours: a systematic review of the nursing literature. J Adv Nurs. 2002;40(3):316-30.

21. Janz NK, Becker MH. The Health Belief Model: a decade later. Health Educ Q. 1984;11:1-47.

22. Rogers RW. A protection motivation theory of fear appeals and attitude change. In: Cacipoppo J, Petty R, editors. Social psychology: A sourcebook. New York: Guilford Press; 1983. p. 153-76.

23. Black JL, Macinko J. The changing distribution of and determinants of obesity in neighborhoods of New York City, 2003-2007. Am J Epidemiol. 2010;171(7):765-75. doi:10.1093/aje/kwp458.

24. Wallach JB, Rey MJ. A socioeconomic analysis of obesity and diabetes in New York City. Prev Chronic Dis. 2009;6(3):1-5. http:// www.cdc.gov/pcd/issues/2009/jul/08_0215.htm.

25. National Institutes of Health. Clinical guidelines on the identification, evaluation, and treatment of overweight and obesity in adults: the evidence report. Bethesda, Maryland: National Heart, Lung, and Blood Institute; 1998.

26. Preacher KJ, Hayes AF. SPSS and SAS procedures for estimating indirect effects in simple mediation models. Behav Res Methods. 2004;36:717-31. doi:10.3758/BF03206553.

27. Faul F, Erdfelder E, Lang A, et al. G*Power 3: a flexible statistical power analysis program for the social, behavioral, and biomedical sciences. Behav Res Methods. 2007;39(2):175-91.
28. Cohen J. A power primer. Psychol Bull. 1992;112(1):155-9.

29. Sivalingam SK, Ashraf J, Vallurupalli N. Ethnic differences in the self-recognition of obesity and obesity-related comorbidities: a crosssectional analysis. J Gen Intern Med. 2011;26:616-20. doi:10.1007/ s1160-010-1623.3.

30. Kramer K, Zinman B, Retnakaran R. Are metabolically healthy overweight and obesity benign conditions? A systematic review and meta-analysis. Ann Intern Med. 2013;159(11): 758-69.

31. Paeratakul S, White MA, Williamson DA, et al. Sex, race/ethnicity, socioeconomic status, and BMI in relation to selfperception of overweight. Obes Res. 2002;10:345-50. doi:10. 1038/oby.2002.48.

32. Dorsey RR, Eberhardt MS, Ogden CL. Racial/ethnic differences in weight perception. Obesity. 2009;17(4):790-5.

33. Stommel M, Schoenborn CA. Variations in BMIX and prevalence of health risks in diverse racial and ethnic populations. Obes (Silver Spring). 2010;18(9):1821-6. doi:10.1038/oby.2009.472.

34. Nguyen NT, Magno CP, Lane KT, et al. Association of hypertension, diabetes, dyslipidemia, and metabolic syndrome with obesity: findings from the National Health and Nutrition Examination Survey, 1999 to 2004. J Am Coll Surg. 2008;207(6):928-34. doi:10.1016/j. jamcollsurg.2008.08.022.

35. August KJ, Sorkin DH. Racial and ethnic disparities in indicators of physical health status: do they still exist throughout late life? J Am Geriatr Soc. 2010;58:2009-15. doi:10.1111/j.1532-5415.2010. 03033.

36. Oyemade U, Rosser P. Development in black children. Adv Behav Pediatr. 1980;1:153-79.

37. DiMatteo MR. Variations in patients' adherence to medical recommendations: a quantitative review of 50 years of research. Med Care. 2004;42(3):200-9.

38. Marshall GN, Hays RD. The Patient Satisfaction Questionnaire Short-Form (PSQ-18). Santa Monica: RAND; 1994. 